\title{
Retraction Note to: Effects of BDNF-Transfected BMSCs on Neural Functional Recovery and Synaptophysin Expression in Rats with Cerebral Infarction
}

\author{
Yongming Zhang ${ }^{1} \cdot$ Binghui Qiu $^{2} \cdot$ Jinbiao Wang $^{1} \cdot$ Yi Yao $^{1} \cdot$ Chunlin Wang ${ }^{1} \cdot$ Jiachuan Liu $^{1}$ \\ Published online: 28 May 2021 \\ (c) Springer Science+Business Media, LLC, part of Springer Nature 2021
}

Retraction Note to: Mol Neurobiol (2017) 54:3813-3824

https://doi.org/10.1007/s12035-016-9946-7

The Editor-in-Chief has retracted this article. Concerns have been raised regarding Fig. 4 that appears to have several duplicated features. The Editor-in-Chief therefore considers the data reported in this the article to be unreliable. The editor was not able to obtain a current email addresses for the authors.

Publisher's Note Springer Nature remains neutral with regard to jurisdictional claims in published maps and institutional affiliations.

The original article can be found online at https://doi.org/10.1007/ s12035-016-9946-7.

Yongming Zhang

zymhf2966@163.com

1 Department of Neurosurgery, No. 105 Hospital of PLA, No. 424 Changjiang West Road, Hefei 230031, Anhui,

People's Republic of China

2 Department of Neurosurgery, Nanfang Hospital, Southern Medical University, Guangzhou 510515, Guangdong,

People's Republic of China 